\title{
Cultura organizacional en el uso de tecnologías de la información y la comunicación en la preparatoria municipal de Tijuana, Baja California, México
}

\author{
María de Jesús Montoya Robles ${ }^{1}$ \\ Gerardo Guillermo León Barrios² \\ https://doi.org/10.36105/stx.2018n1.04
}

\section{Resumen}

Una cultura organizacional para el uso de las tecnologías de la información y la comunicación (TIC) implica no sólo asumir el papel que cada quien representa en la organización, sino también conocer su impacto en el desarrollo educativo o de interacción social de los jóvenes; en este caso, los de la preparatoria municipal de Tijuana. El objetivo de esta investigación es conocer hasta qué punto dichos jóvenes han asumido una cultura hacia el uso de la tecnología, con principal énfasis en internet y redes sociales, por formar esto parte de su vida cotidiana y de los requerimientos de su vida estudiantil y social. Se inicia con una muestra estratificada integrada por 318 estudiantes. Se utiliza el método sumatorio de Likert, y se estima la prueba alfa de Cronbach para conocer la confiabilidad de las respuestas. Los resultados muestran una brecha entre las acciones y una integración hacia una cultura.

\footnotetext{
1 Doctora en Ciencias Administrativas. Profesora investigadora de la Facultad de Humanidades y Ciencias Sociales de la Universidad Autónoma de Baja California. Miembro del Cuerpo Académico Comunicación, Sociedad y Organización. Experiencia docente en áreas de comunicación y organizaciones y métodos cuantitativos. montoya@uabc.edu.mx

2 Doctor en Ciencias Sociales. Profesor investigador de la Facultad de Humanidades y Ciencias Sociales de la Universidad Autónoma de Baja California. Miembro del Cuerpo Académico Comunicación, Sociedad y Organización. Ingeniería en Comunicación Social.gleon@uabc.edu.mx
} 


\section{Palabras clave}

Cultura organizacional, TIC, comunicación, alfabetización digital, educación.

\section{Introducción}

La apropiación de los avances en la tecnología es más evidente en los jóvenes, quienes les dan diversos usos, como la comunicación, el entretenimiento, la socialización y la educación; esto resulta en grandes beneficios, pues varias de esas actividades las pueden hacer de forma paralela, aunque quizá no estén concentrados en ninguna de ellas, o bien, dediquen más tiempo a alguna en particular.

El uso de las TIC es cada vez más generalizado; ya no es privativo de las empresas, pues sus avances han llegado a cada miembro de los diferentes tipos de organizaciones.

Así, toda persona puede estar inmersa en el mundo digital y dejar de lado responsabilidades que se espera que asuma como miembro de una organización, aunque en los extremos existen personas que se han negado al avance tecnológico, limitando su uso al mínimo posible. En la actualidad ambos extremos podrían provocar el aislamiento de las organizaciones de las que se forma parte, además de otras complicaciones propias del papel que la persona desempeña en cada organización, al dejar de hacer actividades por dedicar largas sesiones a entretenimiento o socialización, trabajo, o bien, por acumular largos periodos de ausencia en los medios de comunicación digital. Para asumir una posición hacia las TIC hay que reconocer los cambios en los procesos de interacción de los miembros de las diferentes organizaciones del sistema educativo. Según Raad (2003):

Algunos rasgos sobre la comunicación y la movilización de personas utilizando Internet y TIC y de la cultura digital son: 1) El desplazamiento de los escenarios de socialización: actualmente nos reconocemos y nos relacionamos a través de la televisión y de Internet. Los espacios tradicionales como la plaza, la familia y la escuela han dado paso a estas nuevas tecnologías; 2) El tiempo como experiencia; es decir, vivimos en un tiempo presente en el que estamos interconectados instantáneamente; 3) El fin del espacio como territorio; paralelamente a lo anterior, los espacios ya no son asumidos como locales, sino como interconectados, haciendo que el aquí y ahora tengan nuevos significados; 4) El surgimiento de realidades particulares debido a la atomización del tejido social; y 5) El desdibujar de las fronteras y los límites culturales. (citado en Fernández, 2005, pág. 152).

Las escuelas no son ajenas a este proceso, aun cuando la diversidad de generaciones que las componen pone de manifiesto una brecha en la forma, uso y apropiación de las nuevas 
tecnologías; pese a que algunas escuelas o docentes han dado paso a las TIC en el proceso enseñanza-aprendizaje y pueden verse los usos o abusos, con preocupantes consecuencias, dicho proceso no es el objetivo de esta investigación. Nos ocupa la falta de pertenencia e integración hacia las organizaciones de las que se forma parte; el asumir un aislamiento cuando nuestro entorno está compuesto por nuestras organizaciones.

$\mathrm{Al}$ encontrarse frente a un teléfono, computadora, televisor o cualquier otra TIC, las personas deben estar conscientes de que no dejan de formar parte de varias organizaciones y que sus acciones pueden tener consecuencias en ellas. Así, un estudiante que pasa largas sesiones frente a su computadora en internet en busca de la mejor información para llevar a cabo su tarea podría cumplir con su maestro o con su equipo dentro del grupo, y obtener buenas calificaciones le daría un reconocimiento en su escuela, su familia o comunidad. Pero si el estudiante dedica ese tiempo a socializar en redes o al entretenimiento, podría ser reconocido de manera negativa.

No se trata de vivir con la preocupación de ser juzgado por cada acción que se realice sino de tener conciencia de que no vivimos aislados y formamos parte de varias organizaciones; es decir, lo que hacemos ahora nos puede llevar o alejar de otras organizaciones de las podríamos desear formar parte. Una cultura de uso de las TIC implica tener presente lo anterior, y estar consciente de que hacer o no algo que se espera de uno favorece o perjudica también a otros y aleja de los objetivos o metas personales o de grupo.

Nosnik (2005) propone ocho niveles para desarrollar culturas organizacionales, los cuales sirven de base para la aportación del presente estudio. A partir de su modelo se busca identificar la cultura hacia las TIC desde el individuo, la escuela o la familia, con lo cual se desarrolla el proceso de medición que permitirá conocer, desde la percepción de los estudiantes de la preparatoria municipal de Tijuana, si han desarrollado una cultura hacia el uso de las TIC o resulta una tarea pendiente en alguno de los niveles señalados por el autor.

El sistema educativo municipal de Tijuana cuenta con cinco primarias en las cuales se imparten clases en turno matutino y vespertino; dos secundarias, una de ellas, la Xicoténcatl Leyva Alemán, imparte clases en ambos turnos y la secundaria Adolfo López Mateos sólo cuenta con el turno matutino, ya que comparte sus instalaciones con la escuela preparatoria municipal número 1 Manuel Gómez Morín, en el turno vespertino.

Las escuelas se encuentran en diversas zonas de la ciudad y tienen una población aproximada de 4,652 estudiantes; descartando estudiantes de primero y segundo año de primaria, la población en estudio es de 3,829 . En este avance nos enfocamos a la preparatoria, la cual cuentan con 455 estudiantes, $11.88 \%$ de la población, que se encuentran cursando segundo, cuarto y sexto semestres. Por las características de la preparatoria, al ser la única en el sistema municipal, se levantó una muestra independiente para analizarla por separado 
$y$, considerando que es una generación que puede diferir a los otros niveles educativos, la muestra final fue de 318 estudiantes.

El sistema municipal cuenta en todas sus escuelas con aulas de medios, equipos de cómputo y, en algunos casos, conexión a internet. La preparatoria mencionada cuenta además con biblioteca, y los estudiantes tienen permitido el uso de celular para algunas actividades de clase, entre cada clase, y en el receso.

\section{Las TIC y las organizaciones}

Como todos los adelantos tecnológicos, las TIC llegan a las organizaciones para mejorar procesos con el objetivo de incrementar la producción a partir de los múltiples beneficios que de ellas se esperaban.

Para Sanz (2006), las nuevas tecnologías de la información y la comunicación (NTIC) consistían en computadores, equipos multimedia, redes locales, internet, televisión digital, celulares, entre otros, y las define como sistemas y recursos para la elaboración, almacenamiento y difusión digitalizada de información basada en tecnología informática. Coincide con otros autores al señalar que las NTIC producen profundos cambios y transformaciones de naturaleza social y cultural, además de económicos. Hay que resaltar la consideración del impacto en lo social y cultural que aleja este señalamiento de la concepción de las teorías clásicas de las organizaciones donde los cambios e innovaciones son encaminados a la productividad y los seres humanos son racionales.

Para Brynjolfsson y Hit (2000, citados en Berrío-Zapata y Rojas, 2014), los beneficios de la era de la computación no fueron los esperados o correctamente medidos, o su penetración no se acompañó de los cambios organizacionales necesarios para su aprovechamiento, o su efecto benéfico estaba asociado con activos intangibles sin los cuales su acción se diluía.

Dichos cambios deberían gestionarse en el entorno donde se producen. Así, en las escuelas no se trata sólo de equiparlas con aulas multimedia o salones de computación; no hay que considerar únicamente las cuestiones técnicas sino también la capacitación a los docentes. Tampoco se trata de contar sólo con un profesor de computación, sino de incorporar las TIC en todos los cursos cuando sea posible, de manera que los estudiantes no vean informática o computación como una clase más, sino como un proceso que beneficia y potencia sus posibilidades de conocimiento y productividad en investigaciones o tareas, y que sus profesores se integran a dicho proceso para contribuir en los aprendizajes significativos como producto del acceso a las TIC. 
Para Avgerou (2003, citado en Berrío-Zapata y Rojas, 2014), las organizaciones sinérgicas con las TIC tienden a invertir más en informática, sofisticando su gestión permanentemente y alejándose rápidamente de sus competidores. Pero indica que, en contextos no industriales, las sociedades y organizaciones no informatizadas mantienen sus rutinas tradicionales porque enfrentan simultáneamente la absorción de técnicas e instrumentos y la necesidad de emular las idiosincrasias y rutinas de sus entornos de origen, situación a la que se enfrentan algunos grupos o escuelas.

Lo anterior es comprensible pues hay una brecha generacional de acceso a las TIC entre profesores y estudiantes, la cual a veces es muy amplia y otras veces muy pequeña, con los jóvenes docentes, aunque algunos profesores muestran que no hay edad para aceptar el reto hacia la adopción de la tecnología.

Para Berrío-Zapata y Rojas (2014), el cambio se percibe como costoso, demorado y arriesgado, lo cual facilita actitudes tecnofóbicas, indiferentes o de aceptación por simple moda. Con esto es posible adentrarnos en el aspecto cultural, ya que la percepción y forma de uso de las TIC genera una barrera o brecha digital, tangible o no, que puede ser entre países, organizaciones o individuos; la cual depende de la actitud y la frontera de posibilidades con las que se les asume.

\section{Culturas organizacionales y TIC}

Dado que el concepto de cultura puede verse desde diferentes ángulos, precisamos que nos referimos principalmente a las convenciones que estructuran la vida cotidiana ante las diversas actividades que se desarrollan en las organizaciones. Como menciona García Ruiz:

La cultura incluye tanto un sistema compartido de respuestas como un diseño social de la conducta individual. Para saber si un rasgo de la vida de un grupo es cultural, se pueden atender a tres características intrínsecas a la cultura:a) si se comparte socialmente; b) si se transmite entre generaciones; c) si se requiere aprendizaje, pues la cultura no es innata, sino que se adquiere mediante procesos de socialización (2013, p. 305).

Así observamos cómo las interacciones de cada día en las organizaciones van dando forma a una cultura y a la manera en que se aprende. Bajo condiciones favorables los colaboradores comparten sus conocimientos entre ellos, y en algunos casos con usuarios como los padres de familia o tutores, o el sistema educativo municipal. Por lo tanto, se esperaría que las or- 
ganizaciones y sus colaboradores desarrollen cultura de forma paralela y en beneficio de la escuela como organización.

Para García (2013), las organizaciones no son únicamente instrumentos para el logro de metas. Son también sistemas sociales en los que sus miembros desarrollan vínculos recíprocos a partir de las tareas que desempeñan. Son comunidades a escala que desarrollan una cultura propia. Así, la cultura de una organización es un conjunto de creencias y valores compartidos por sus miembros; los cuales se manifiestan en sus procedimientos, actitudes y convenciones básicas, que están en la base de sus estructuras y sistemas de gestión, así como en las actividades propias de la vida cotidiana.

Por su parte, Cújar, Ramos, Hernández y López (2013), Nosnik (2005) y García (2013) comentan que la cultura organizacional se empezó a gestar desde la propia teoría administrativa, pero su evolución llevó al término de la cultura organizacional al contemplar a las personas no sólo como un ser racional sino como un ser social.

Nosnik (2005) presenta dos esquemas conceptuales, que servirán de base en el documento para comprender el cambio en la teoría del ser racional al social, para definir el avance o evolución del pensamiento administrativo. El primer esquema es el propuesto por Jablin, quien dice que el pensamiento administrativo ha pasado por cuatro etapas: la teoría clásica, la teoría humanista, la teoría de sistemas y la teoría contingente. Nosnik encuentra semejanzas entre el esquema anterior y el planteado por Peter y Waterman, los cuales aunque explicitan las diferencias entre etapas, enfatizan las variables: actores y sistemas, para su explicación.

Para Peter y Waterman, la historia de la Administración también se divide en cuatro etapas: la primera concibe a las personas como actores racionales en un contexto organizacional cerrado, es decir, con poca o nula consideración del intercambio de energía con el medio ambiente; la segunda, que considera a las personas como actores sociales en contextos cerrados; la tercera, que avanza de una concepción de sistemas cerrados a sistema abierto respecto de la organización y, sin embargo, regresa a una concepción de actores racionales. Y finalmente, la cuarta, que está vigente, en la cual existen organizaciones como sistemas abiertos habitadas por actores sociales más que racionales (Nosnik, 2005, p. 17).

Nosnik (2005) aclara la concepción de actor racional, que consiste en considerar la naturaleza humana como caracterizada por un interés y una capacidad de normar su vida institucional por medio de la toma de decisiones, es decir, el control por medio de la racionalidad y la optimización de los recursos a su alcance para cumplir con los objetivos planteados. 
Así, en un escenario donde las TIC son una opción, el ser racional busca beneficios materiales económicos, mientras que el ser social se preocupa por sí mismo, pero también por cambiar lo que sus acciones causan a los demás en su entorno; de esta manera los medios y las tecnologías asumen un nuevo papel al facilitar procesos de interacción y desarrollo laboral.

De acuerdo con Aguaded (2005), los medios y las tecnologías han dejado de ser meras herramientas preparadas para servir a quienes las usan, para convertirse en parte del sistema cultural que las acoge. Sancho (1994, citado en Aguaded, 2005) afirma que el tema no es que las máquinas hayan tomado el mando, sino que al decidir utilizarlas realizamos muchas elecciones culturales implícitas. La tecnología no es un simple medio, sino que se ha convertido en un entorno y una forma de vida: éste es su impacto sustantivo.

Por otra parte, Berrío-Zapata y Rojas (2014) encontraron que los estudiantes emplean las TIC pero, dada su formación y educación, no se apropian de sus posibilidades productivas más allá de los usos básicos o recreativos. Además, mencionan que la apropiación tecnológica es un fenómeno individual, pero también colectivo, que incluye factores políticos, económicos y culturales que deben ser analizados en conjunto.

En el caso de los estudiantes es conveniente conocer si hay una cultura que de manera consciente oriente sus procesos de interacción mediados por tecnologías, o en sus usos, y si se desarrolla una cultura organizacional.

\section{De la cultura organizacional a la cultura de las TIC}

El cambio se refiere no sólo a una actitud o a modificar comportamientos, sino a asumir los cambios de acuerdo con el papel que se desempeña como miembro en las diversas organizaciones. Un estudiante que requiere comunicarse con su profesor para aclarar una duda puede decidir hacerlo de forma inmediata, quizá asincrónica, utilizando algún medio, o esperar ver a su profesor cara a cara uno o varios días después; mientras tanto su trabajo se habrá retrasado o habrá avanzado dependiendo de su decisión y del tiempo de respuesta de su profesor, quien también debe asumir su papel en la organización. De lo contrario, las brechas se abrirán al no adoptar el papel de las TIC o abrirse el espacio para escuchar al estudiante.

Nosnik (2005) propone ocho etapas para el desarrollo cultural de la organización. Dada la fuerte relación de la organización en el desarrollo de las TIC, tomamos de base su modelo teórico para el diseño del instrumento que permite conocer el desarrollo de la cultura hacia las TIC por parte de los jóvenes. La base de este modelo es la obra de Erik H. Erickson El ciclo vital completado (Erikson, 1985) y la obra La metodología de los programas científicos de investigación de Imre Lakatos (1975), (Nosnik, 2005 p. 118). En resumen: 
Para Nosnik, (2005) la etapa ocho que consiste en la integración de las siete anteriores y busca la calidad de vida de los miembros de la organización, para lograrlo debe contar con un líder sabio, que sea moral y experimentado. Así cada responsable de un equipo de trabajo debe apoyar a sus colaboradores (etapa I); brindarles condiciones de libertad para desarrollarse y ser capaces de lograr sus propios objetivos (etapa II); promover la participación en el trabajo (etapa III); ser emprendedor (etapa IV); tener una visión global y de competencia de los principales mercados o ámbitos de la sociedad donde se ubica su organización (etapa V); ser capaz de cambiar y revitalizar con el cambio su entorno (etapa VI) y por último, actuar responsablemente en su sociedad a favor de generaciones futuras (etapa VII).

En el siguiente apartado se establece la relación de preguntas con cada etapa propuesto por Nosnik, aplicado a las TIC en el entorno de la escuela. Aquí se presenta brevemente su significado:

I. Unidad. Cada colaborador debe tener confianza en sí mismo y en sus líderes para lograr las tareas, metas y objetivos de su puesto, y estos últimos deben crear condiciones para que eso suceda.

II. Capacidad. El colaborador demuestra lo que es, lo que sabe y lo que sabe hacer en un ambiente que se lo facilita.

III. Consenso. El valor central es el acuerdo y el segmento focal el equipo, desde el puesto más alto al de menos estatus, con un liderazgo participativo, esto es, abierto a la comunicación.

IV. Efectividad. Una cultura organizacional efectiva resulta de la motivación y capacidad mostrada de sus miembros, demanda un líder emprendedor, individuos con iniciativa, perseverancia, tenacidad, resistencia a la frustración y un alto nivel de energía en el trabajo.

V. Competencia. Busca una posición de liderazgo en el ámbito de la sociedad a la que se sirve.

VI. Responsabilidad institucional. Nosnik resalta tres categorías: i. la responsabilidad social: cumplir las metas y objetivos que sirven a la misión; ii. la responsabilidad comunitaria: como organización, reconocer ser parte de una comunidad y, en la medida de sus posibilidades, recursos y la función comunitaria de su competencia, favorecer el desarrollo de la misma a través de las consecuencias positivas de su desempeño; y iii. la responsabilidad política: cuidar e incorporar en su visión los aspectos de contribuir al bien común.

VII. Ecología. La responsabilidad que tienen las organizaciones de disminuir el deterioro del medio ambiente en las ciudades y urbanizaciones, y concientizar sobre la amenaza tan grande que esto representa para el futuro de todos. 
VIII. Integración. Consiste en un esquema de etapas consecutivas e incrementales de desarrollo cultural; es integrar los siete niveles anteriores. Para Nosnik el concepto de calidad de vida sintetiza dichas etapas. Busca que la vida organizacional de las personas, en cuanto profesionales, contribuya a su propio bienestar y al de su organización, comunidad, sociedad y mundo.

\section{Metodología}

Considerando un nivel de confianza de $95 \%$-ya que la muestra es representativa de la población, es decir, tiene las características de la población-y un margen de error de 3\% sobre la validez de los resultados - previendo la posibilidad de la no respuesta, la falta de interés o la desconfianza al contestar por parte los estudiantes-, se utiliza la fórmula para determinar un tamaño de muestra de una población finita Fisher y Navarro (1990):

$$
n=\frac{\sigma^{2} N p q}{e^{2}(N-1)+\sigma^{2} p q}
$$

Donde:

$\sigma$ es el coeficiente de confianza $=(1.96)^{2}$ área bajo la curva $=0.4750$

$p \quad$ es la probabilidad de éxito $=0.50$

$q$ es la probabilidad de fracaso $=0.50$

$e \quad$ es el error de estimación $=3 \%=(0.03)^{2}=0.0009$

$N$ es el tamaño de la población $=455$

$n$ es el tamaño de muestra $=320$ (la muestra final quedo en 318 estudiantes, que fueron quienes contestaron correctamente el cuestionario).

Hernández, Fernández y Baptista (2010) indican que la prueba Alfa de Cronbach se utiliza para corroborar la coherencia o consistencia interna del cuestionario donde, de acuerdo a su resultado, nos indica la confiabilidad del instrumento; si el resultado de la prueba es de 0.25 en la correlación o coeficiente, indica una baja confiabilidad; si el resultado es de 0.50 , es media o regular; en cambio, si se supera a 0.75 es aceptable, y si llega a 0.90 es muy elevado el nivel de confiabilidad del instrumento.

De acuerdo con los resultados obtenidos a través del programa de SPSS (Statistical Package for the Social Sciences), se encuentra que la confiabilidad de las preguntas es muy 
elevada, el valor mínimo es de 0.925 y llega hasta 0.930 . La estadística de confiabilidad como instrumento es de 0.929 en conjunto.

\section{Método sumario de Likert:}

Se aplicará el método sumario estimando los puntajes por cada ítem, por etapa y posteriormente por el puntaje general; dicho puntaje se obtiene de multiplicar el número de personas que contestaron las preguntas y multiplicándolo primero por las posibilidades de puntaje de acuerdo a los cinco niveles de los elementos tipo Likert: (n)(k), donde $n$ es el número de cuestionarios, pero al no obtener las respuestas de todos los integrantes, éste varía, y $k$ es la cantidad de alternativas, primero otorgando un cinco a "Totalmente de acuerdo", un cuatro a "De acuerdo", un tres a "Ni de acuerdo ni en desacuerdo", un dos a "En desacuerdo" y un uno a "Totalmente en desacuerdo".

Con lo anterior se establecerán los puntajes deseados. Para obtener los puntajes resultantes se multiplicará cada posible respuesta 1,2, 3, 4 o 5 por su frecuencia; la suma total ubicará las respuestas e indicará el resultado para cada pregunta, y la suma de preguntas indicará los niveles para cada etapa de desarrollo cultural de acuerdo con el modelo de Likert, como se muestra en la Tabla 1. La etapa ocho se obtendrá de la suma de las etapas anteriores y sus resultados.

La relación establecida entre las etapas del desarrollo cultural de las organizaciones y el desarrollo cultural de las TIC se presenta a continuación:

TABLA 1. ETAPAS DEL DESARRO $O$ O OUIUURAL DE LAS TIC EN ESTUDIANTES

\begin{tabular}{|l|l|}
\hline Etapa cultural Nosnik & $\begin{array}{l}\text { 1. Elementos tipo Likert utilizados } \\
\text { 2. Cuando uso las TIC... }\end{array}$ \\
\hline Unidad & $\begin{array}{l}\text { 3. Lo hago de manera apropiada/responsable fuera de la escuela. } \\
\text { 4. Lo hago de manera apropiada/responsable dentro de la escuela. } \\
\text { 5. Mis maestros o directivos de la escuela me recuerdan el adecuado uso. } \\
\text { 6. Mis padres y familiares me recuerdan el adecuado uso. }\end{array}$ \\
\hline Capacidad & $\begin{array}{l}\text { 7. Aprendo en internet la forma apropiada/responsable de utilizarlas. } \\
\text { 8. Realizo primero tareas o proyectos escolares relacionados con las TICantes de } \\
\text { navegar en internet, jugar o socializar. } \\
\text { 9. Cuando el profesor me deja una tarea, por ejemplo una investigación o un vi- } \\
\text { deo, en donde tengo que usar las TIC, me explica cómo realizarla, qué páginas } \\
\text { consultar y las características que debe tener. }\end{array}$ \\
\hline
\end{tabular}




\begin{tabular}{|c|c|}
\hline Consenso & $\begin{array}{l}\text { 10. Mis padres o familiares cercanos revisan lo que hago o consulto en las TIC. } \\
\text { 11. Mis padres o familiares me ayudan a distribuir el tiempo que les dedico a las TIC. } \\
\text { 12. Mis padres o familiares, maestros o compañeros me limitan el tiempo de uso. } \\
\text { 13. Mis padres me explican para qué se debe utilizar internet. } \\
\text { 14. Mis maestros me explican para qué se debe utilizar internet. }\end{array}$ \\
\hline Efectividad & $\begin{array}{l}\text { 15. Me gusta distribuir mi tiempo para desarrollar las actividades que se espera } \\
\text { que realice. } \\
\text { 16. El uso apropiado de las TIC favorece el trabajo en equipo. } \\
\text { 17. Encuentro soluciones para llevar a cabo mi tarea aun cuando de momento no } \\
\text { tenga acceso a las TIC. } \\
\text { 18. Si no encuentro una tarea en internet, sigo navegando hasta encontrar infor- } \\
\text { mación. } \\
\text { 19. Si tengo tarea de internet, reviso diversas páginas y selecciono la mejor infor- } \\
\text { mación. }\end{array}$ \\
\hline Competencia & $\begin{array}{l}\text { 20. Mi grupo cuenta con un grupo o acceso web para comunicarse. } \\
\text { 21. Si hay una actividad en equipo me informo de lo que me corresponde hacer. } \\
\text { 22. Mis profesores o directivos utilizan las TIC para informar sobre asuntos urgen- } \\
\text { tes, como suspensión de clases, juntas, becas. } \\
\text { 23. Mis profesores utilizan las TIC para enviarnos tarea o actividades escolares. }\end{array}$ \\
\hline Responsabilidad institucional & $\begin{array}{l}\text { 24. Lo que publico o comento en redes sociales puede tener impacto en mi grupo } \\
\text { y escuela. } \\
\text { 25. Lo que publico o comento en redes sociales puede tener impacto en mi familia. } \\
\text { 26. Me gustaría enseñar a mis familiares, compañeros y amigos el adecuado uso } \\
\text { de las TIC. } \\
\text { 27. Me gustaría contribuir a crear conciencia sobre el buen uso de las TIC. } \\
\text { 28. Si todos hacemos uso adecuado de ellas evitamos problemas sociales. }\end{array}$ \\
\hline Ecología & $\begin{array}{l}\text { 29. Las TIC son una herramienta que permite difundir temas para crear conciencia } \\
\text { ecológica, educativa y social. } \\
\text { 30. En redes sociales me desagradan los comentarios con contenido violento. } \\
\text { 31. Mis compañeros y yo utilizamos un lenguaje fuerte o soez por diversión. }\end{array}$ \\
\hline
\end{tabular}

FUENTE: EABORACIÓN PROPIA.

\section{Resultados}

Las puntaciones obtenidas, reunidas en la Tabla 2, demostraron que los jóvenes están de acuerdo o totalmente de acuerdo con el uso de las tecnologías de la información y la comunicación, aun cuando las puntuaciones de Likert resultaron altas, de manera particular las correspondientes a las etapas de Unidad, Efectividad y Competencia. 
En contraparte, los puntajes más bajos, aun cuando se encuentran en el intervalo de “De acuerdo", están próximos al nivel neutro de Likert; estos niveles son Capacidad, el más bajo, seguido del nivel de Ecología, Consenso y, por último, Responsabilidad institucional.

TABLA 2. RESULTADOS DEL MÉTODO SUMARIO DE UKERT, PUNTUACONES ÓPTIMASY OBTENIDAS

\begin{tabular}{|c|c|c|}
\hline \multicolumn{3}{|c|}{ I. UNIDAD } \\
\hline I NFE RIOR & SU P E RIOR & RE SU LTADO \\
\hline 0 & 1166 & \\
\hline 1167 & 2332 & \\
\hline 2333 & 3498 & \\
\hline 3499 & 4664 & \\
\hline 4665 & 5830 & 4768 \\
\hline
\end{tabular}

\begin{tabular}{|c|c|c|}
\hline \multicolumn{3}{|c|}{ V. COM P ETE N CIA } \\
\hline I N FE RIOR & SU P E RIOR & RE SU LTADO \\
\hline 0 & 1182 & \\
\hline 1183 & 2364 & \\
\hline 2365 & 3546 & \\
\hline 3547 & 4728 & \\
\hline 4729 & 5910 & \\
\hline
\end{tabular}

\begin{tabular}{|c|c|c|}
\hline \multicolumn{3}{|c|}{ II. CAP ACIDAD } \\
\hline IN FE RIOR & SU P E RI OR & RE SU LTADO \\
\hline 0 & 908 & \\
\hline 909 & 1816 & \\
\hline 1817 & 2724 & \\
\hline 2725 & 3632 & 3608 \\
\hline 3633 & 4540 & \\
\hline
\end{tabular}

\begin{tabular}{|c|c|c|}
\hline \multicolumn{3}{|c|}{ VI. RE SP ON SA B I UDAD IN STITU CI ON A L } \\
\hline I N FE RI OR & SU P E RI OR & RE SU LTAD O \\
\hline 0 & 1366 & \\
\hline 1367 & 2732 & \\
\hline 2733 & 4098 & \\
\hline 4099 & 5464 & 5078 \\
\hline 5465 & 6830 & \\
\hline
\end{tabular}

\begin{tabular}{|c|c|c|}
\hline \multicolumn{3}{|c|}{ III . CONSE N SO } \\
\hline IN FE RI OR & SU P E RI OR & RE SU LTADO \\
\hline 0 & 1280 & \\
\hline 1281 & 2560 & \\
\hline 2561 & 3840 & \\
\hline 3841 & 5120 & \\
\hline 5121 & 6400 & \\
\hline
\end{tabular}

\begin{tabular}{|c|c|c|}
\hline \multicolumn{3}{|c|}{ VII. E COLOGIA } \\
\hline INFERIOR & SUP E RIOR & RE SU LTADO \\
\hline 0 & 855 & \\
\hline 856 & 1710 & \\
\hline 1711 & 2565 & \\
\hline 2566 & 3420 & \\
\hline 3421 & 4275 & 3295 \\
\hline
\end{tabular}

\begin{tabular}{|c|c|c|}
\hline \multicolumn{3}{|c|}{ IV. E FE CTIVIDAD } \\
\hline I N FERIOR & SU P E RIOR & RE SU LTADO \\
\hline 0 & 1515 & \\
\hline 1516 & 3030 & \\
\hline 3031 & 4545 & \\
\hline 4546 & 6060 & \\
\hline 6061 & 7575 & 6144 \\
\hline
\end{tabular}

\begin{tabular}{|c|c|c|}
\hline \multicolumn{3}{|c|}{ VIII . INTE GRACIÓN } \\
\hline I N FE RI OR & SU P E RIOR & RE SU LTADO \\
\hline 0 & 8272 & \\
\hline 8279 & 16544 & \\
\hline 16551 & 24816 & \\
\hline 24823 & 33088 & 32020 \\
\hline 33095 & 41360 & \\
\hline
\end{tabular}

FUENTE: EABORACÓN PROPIA. 
Los jóvenes de la preparatoria indicaron el tiempo que dedican en un día regular a internet: en promedio seis y media horas con una desviación estándar alta cercana a las cuatro horas; esto se explica porque los estudiantes tienen acceso a teléfonos inteligentes durante clases, aun cuando algunos señalaron que no utilizan ningún dispositivo en dicho horario, mientras que pocos indicaron que lo usan todo el tiempo, lo cual no se incluyó en el cálculo de la media para evitar sesgo en el promedio.

\section{Conclusiones}

Pareciera no existir una brecha entre la apropiación de una cultura organizacional; los jóvenes saben lo que está bien y aparentemente les gustaría adoptar dicha cultura, pero las horas que destinan a las TIC o a internet indican que todavía no lo han contemplado como algo necesario, lo cual aplica no sólo a los jóvenes, sino también a los docentes, padres de familia y administrativos.

Sorprende el hecho de que el nivel II, Capacidad, sea uno de los más bajos cuando la actitud debe tender a encargarse de sus tareas y conocer el correcto uso de las tecnologías. El siguiente en puntaje reducido, el nivel de la Ecología, por los aspectos negativos debería obtener puntajes más bajos, entre 1 y 2 , pues habla de los mensajes violentos o con lenguaje soez. Otro nivel es el Consenso, el III, que trata de la interacción que permite el uso consciente o buen uso de las TIC. Por último, la Responsabilidad institucional repercute no sólo al exterior, sino en cada miembro, ya que lo que se publique en redes sociales puede afectar a la familia o a los compañeros, o bien, contribuir a un mejor uso de las TIC.

Para Fernández (2005), una de las formas de retomar el contexto de las tecnologías que han hecho crecer una brecha entre las organizaciones y sus miembros al asumir las TIC "es tender al llamado fetichismo tecnológico, en el que el cambio de paradigma cultural estaría resuelto con la sola adquisición de tecnologías, sin tener en cuenta aspectos y necesidades sociales ni visiones a largo plazo". (p. 78).

Esta visión es algo que se debe abandonar en las instituciones educativas; el entendimiento de los beneficios y los problemas que pueden ocasionar las TIC contribuirá a adoptar y asumir, a través de una cultura hacia los medios y la tecnología, el impacto en la vida cotidiana, la escuela o con los amigos; ese entendimiento permitirá a los miembros regular sus acciones de forma natural, coadyuvar a sus interacciones y mejorar su comunicación, sea o no mediada tecnológicamente. 


\section{Referencias}

Aguaded, J. I. (2005). Estrategias de edu-comunciación en la sociedad audiovisual. Comunicar, 24, $25-34$.

Berrío-Zapata, C. \& Rojas, H. (2014). La brecha digital universitaria: La apropiación de las TIC en estudiantes de educación superior en Bogotá (Colombia). Comunicar. Revista Científica de Educomunicación, XXII(43), 133-142.

Cújar, A. D., Ramos, C. D., Hernández, H. E. \& López, J. M. (2013). Cultura organizacional: evolución en la medición. Estudios gerenciales. Elsevier, 29, 350-355.

Erikson, E. (1985). El ciclo vital completado. Bueno Aires: Paidós.

Fernández, C. C. (2009). La comunicacion en las organizaciones. México: Trillas.

Fernández, F.J. (2005). Brecha e inclusión digital en Chile: los desafíos de una nueva alfabetización. Comunicar, 24, 77-84.

Fisher, L. \& Navarro, A. (1990). Introducción a la investigación de mercados. México: McGraw-Hill.

García, R. P. (2013). Cultura de la organización. En M. A. Lucas, Sociología de las organizaciones. Influencia de las tecnologías de la información (pp. 301-322). Madrid: Fragua.

Hernández, R., Fernández, C. \& Baptista, P. (2010). Metodología de la investigación. Perú: McGraw-Hill.

Lakatos, I. (1978). La metodología de los programas de investigación científica. Madrid: Alianza Universidad.

Levine, D., Krehbiel, T. \& Berenson, M. (2006). Estadística para administración. México: Pearson Educación.

Martínez, V. A. (2008). Comunicación organizacional práctica. Manual gerencial. (2a. ed.). México: Trillas.

Nosnik, A. (2005). Culturas organizacionales: origen, consolidación y desarrollo. España: Netbiblo. 